\title{
Switching From High Doses of Opioids to Tapentandol PR: Two Cases of Neuropathic Pain - Cancer and Non-Cancer
}

Vincenzo Pota1, Maria Caterina Pace ${ }^{1}$, Maria Beatrice Passavanti ${ }^{1}$, Pasquale Sansone ${ }^{1}$, Manlio Barbarisi ${ }^{2}$ and Caterina Aurilio ${ }^{1 *}$

${ }^{1}$ Department of Anaesthesiological, Surgical and Emergency Sciences, Second University of Naples, Italy

${ }^{2}$ Neurosurgery Unit, Second University of Naples, Italy

\begin{abstract}
Opioids represent the main treatment for moderate to severe chronic pain both oncological than neuropathic one. They are assuredly effective drugs to relieve the pain symptoms but are burdened in chronic use by adverse events that may limit their use (tolerance, nausea, vomiting, itching, sleepiness). The strategy of switching opioids is a response to this. Rotation from one opioid to another, regulated by conversion tables, is useful to ensure a better balance of analgesia and toxicity. Tapentadol is a novel, centrally acting analgesic with dual mechanism of action, combining mu-opioid receptor agonism with noradrenaline reuptake inhibition in the same molecule. It has an improved side effect profile when compared to opioids and nonsteroidal anti-inflammatory drugs. The dual mechanism of action makes Tapentadol a useful analgesic to treat acute, chronic, and neuropathic pain. As the Pain Treatment Service of the Second University of Naples, we present two clinical cases of patients being treated with high dose opioids who we rotated to tapentadol.
\end{abstract}

Keywords: Tapentadol; Pain therapy; Opioid switching

\section{Case 1}

A 54-year-old man who had had adenocarcinoma of the pancreas 3 years ago, on whom a cephalopancreasectomy had been performed, followed by adjuvant chemotherapy, came to our Pain treatment Service. Two years after the operation he was diagnosed with a secondary tumour due to repetitive processes in the lungs. Furthermore, a biopsy of the very painful and in some cases ulcerated subcutaneous nodules in the upper limbs and scalp revealed that they were secondary repetitive processes of the pancreatic adenocarcinoma. The patient also presented type II diabetes mellitus (treated with a hypoglycaemic diet, metformin $1000 \mathrm{mg}$ capsules at meals and 10 I.U. of lantus insulin at night).

For the pain caused by the repetitive processes, he had been treated with different NSAIDs (Nonsteroidal anti-inflammatory drugs), weak and strong opioids, transdermal opioids and transmucosal fentanyl for the treatment of breakthrough pain, reaching such high doses as to cause the onset of severe side effects. The last treatment carried out had been oxycodone $80 \mathrm{mg} /$ naloxone $40 \mathrm{mg}$ /day and oxycodone 40 $\mathrm{mg} /$ day.

On admission his pain scores were assessed as NRS (Neuropathic Rating Scale) $=9 / 10$, DN4 (Douleur Neuropathique en 4 questions) $=8$.

He started treatment with pregabalin $150 \mathrm{mg} /$ day for seven days followed by $300 \mathrm{mg} /$ day and treatment was switched from the high dosage opioids the patient was taking to tapentadol PR (Prolonged Release), administered at a dosage of $400 \mathrm{mg} /$ day, increased to $500 \mathrm{mg} /$ day after 8 days. At the end of the switch the patient displayed a notable diminution in pain intensity, $\mathrm{NRS}=2 / 10, \mathrm{DN} 4=3$. This improvement in symptoms remained constant for over three months, with no significant side effects.

\section{Case 2}

A 67-year-old woman, weighing $56 \mathrm{~kg}$, suffering with chronic lower back pain for the previous 5 years, exacerbated after two kyphoplasty procedures. The patient also suffered from arterial hypertension, chronic atrial fibrillation, prior aorto-subclavian bypass and COPD (Chronic obstructive pulmonary disease). She was being treated with: calcium antagonists, dicumarols, bronchodilators and steroids as required. The reported mean weekly VAS (Visuoanalogic Scale) pain score was $90 \mathrm{~mm}$, with DN4=7/10 and S.F. McGill (Short Form McGill
Pain Questionnaire) $=24$, and she presented pain evoked by movement with approximately 5 exacerbations per hour.

\section{Creatinine $0.97 \mathrm{mg} / \mathrm{dl}$ with creatinine clearance $44 \mathrm{ml} / \mathrm{min}$.}

She took the treatment prescribed by her general practitioner, as follows: oxycodone $20 \mathrm{mg}$ as needed with a mean daily dose of $80 \mathrm{mg}$, escitalopram and ketorolac $30 \mathrm{mg} \mathrm{fl}$ i.m. as needed, with a mean daily dose of $60 \mathrm{mg}$, and she is used to assume benzodiazepine for sleeping.

The switch was carried out at her first appointment in our clinic, where she was prescribed tapentadol PR $200 \mathrm{mg}$ /day and pregabalin $150 \mathrm{mg} /$ day. She was advised to decrease the escitalopram and ketorolac, and to progressively reduce the benzodiazepines. After 7 days the observed VAS score was $70 \mathrm{~mm}$ with a mean weekly VAS of $60 \mathrm{~mm}, \mathrm{DN} 4=3 / 10$ and S.F.McGill=10. Tapentadol PR $400 \mathrm{mg} /$ day and pregabalin $150 \mathrm{mg} /$ day were prescribed. After 14 days the mean VAS appeared to have reduced further to $<40 \mathrm{~mm}$ and the DN4 was $2 / 10$. The patient had stopped taking benzodiazepine and antidepressants and the HADS (Hospital Anxiety and Depression Scale) questionnaire highlighted the absence of depressive symptoms. After six months of treatment no increase in dosage was necessary, because control of the pain symptoms was maintained constant with negligible side effects, so much so that the patient was able to start a cycle of physiotherapy.

\section{Discussion}

The purpose of this article was to describe two clinical cases in that drug switch of tapentadol resulted in improvement of pain condition and side effects of patients who had been treated with other opioid drugs. The first case is a patient with neuropathic pain associated with cancer

*Corresponding author: Caterina Aurilio, Department of Anaesthesiological Surgical and Emergency Sciences, Second University of Naples, Italy, Tel: 081 5665215; E-mail: caterina.aurilio@unina2.it

Received May 21, 2014; Accepted July 23, 2014; Published July 25, 2014

Citation: Pota V, Pace MC, Passavanti MB, Sansone P, Barbaris M, et al (2014) Switching From High Doses of Opioids to TapentandolPR: Two Cases of Neuropathic Pain-Cancer and Non-Cancer. J Clin Case Rep 4: 394 doi:10.4172/2165-7920.1000394

Copyright: (c) 2014 Pota V, et al. This is an open-access article distributed under the terms of the Creative Commons Attribution License, which permits unrestricted use, distribution, and reproduction in any medium, provided the original author and source are credited. 
pain, and the second one, chronic lumbosciatic pain with radiculopathy. Both patients were switched to tapentadol from high doses of opioids that in case 2 were also being taken improperly. Switching opioids is a useful strategy in the long-term treatment of chronic pain, when tolerance phenomena appear or when adverse events limit the use of opioids, ruining the patient's quality of life [1,2]. The tolerance may be associated with both pharmacokinetic and pharmacodynamic processes. This is the context for our interest in switching to tapentadol PR. Tapentadol (3-((1R,2R)-3-(dimethylamino)-1ethyl2methylpropyl)phenolhydrochloride) is a non-racemic molecule. The molecular formula of Tapentadol is $\mathrm{C}_{14} \mathrm{H}_{23} \mathrm{NO} . \mathrm{HCl}$. Tapentadol provides analgesia through two mechanisms of action, i.e., MOR (muopioid receptor) agonism and noradrenaline reuptake inhibition (NRI) $[3,4]$. It binds to MOR selectively, with a more than or equal to tenfold affinity compared with delta- and kappa- opioid receptors. The affinity of Tapentadol for MOR is 44-fold lower than that of morphine [5]. NRI increases levels of noradrenaline (NA), which in turn leads to analgesia through activation of inhibitory alpha-2 receptors. This dual mechanism of action contributes to its opioid sparing effects [6]. Tapentadol is also a weak serotonin reuptake inhibitor, but this action does not contribute to its analgesic effect [7]. The analgesic potency of Tapentadol is only 2-3 times lower than that of morphine, despite its lower affinity of binding to MOR; this is due to additional noradrenergic mechanism of action of the drug [8].

The dual mechanism guarantees not only efficacious analgesia for a broad range of acute and chronic pain pathologies, but also an effective saving on the equianalgesic dose [3,4]. Moreover the onset of adverse events correlated with the use of an opioid should be less. Furthermore, since it does not have serotoninergic properties, tapentadol does not present adverse events correlated with the increase in serotonin in the central and enteric nervous system (constipation, nausea, vomiting, diarrhoea).

Another interesting aspect of this case report is the switching of opioids in subjects with multiple comorbidities, who are, therefore, being treated with multiple drugs. The choice of tapentadol is also based on its metabolism. $97 \%$ of tapentadol is metabolised through glucuronation in the liver, less than $3 \%$ by the CYP450 (Cytocrome P450) isoenzymes, and its administration in subjects being treated with multiple drugs may therefore be considered safer $[5,6]$. The use of pregabalin in association with the opioid derives both from the attention paid by our centre to the EFNS guideline, which proposes gabapentinoids as drugs of first choice in neuropathic pain, and from our experience of the multimodal treatment of chronic neuropathic pain $[7,8]$.

The two clinical cases presented show good control of the pain symptoms from the start of the switch, and this control is maintained for a long time, in patients who previously took high dosage opioids. So tapentadol could be a treatment option in patients with chronic neuropathic pain in treatment with opioids that have developed tolerance or adverse events. Obviously, this single case report should be considered a prompt for the scheduling of a randomised clinical trial to establish suitable equianalgesic tables and indications for the switch to tapentadol [9-14].

\section{References}

1. Mercadante S, Valle A, Porzio G, Fusco F, Aielli F, et al. (2013) Opioid switching in patients with advanced cancer followed at home. A retrospective analysis. J Pain Symptom Manage 45: 298-304.

2. Aurilio C, Pace MC, Pota V, Sansone P, Barbarisi M, et al. (2009) Opioids switching with transdermal systems in chronic cancer pain. J ExpClin Cancer Res 28: 61.

3. Afilalo M, Stegmann JU, Upmalis D (2010) Tapentadol immediate release: a new treatment option for acute pain management. J Pain Res 3: 1-9.

4. Bee LA, Bannister K, Rahman W, Dickenson AH (2011) Mu-opioid and noradrenergic $\hat{I} \pm(2)$-adrenoceptor contributions to the effects of tapentadol on spinal electrophysiological measures of nociception in nerve-injured rats. Pain 152: $131-139$.

5. Tzschentke TM, Jahnel U, Kogel B, Christoph T, Englberger W, et al. (2009) Tapentadol hydrochloride: a next-generation, centrally acting analgesic with two mechanisms of action in a single molecule. Drugs Today (Barc) 45: 483496.

6. Hartrick CT, Rozek RJ (2011) Tapentadol in pain management: a î1/4-opioid receptor agonist and noradrenaline reuptake inhibitor. CNS Drugs 25: 359-370.

7. Cristoph T, De Vry J, Jahnel U, Tzschentke TM (2009) Anti-allodynic activity of tapentadol in a rat model of neuropathic pain depends on opioid and noradrenergic, but no serotonergic, mechanisms. Eur J Pain 13:205.

8. Tzschentke TM, De Vry J, Terliden R (2009) Tapentadol hydrochloride: Analgesic mu-opioid receptor agonist noradrenaline reuptake inhibitor. Drugs Today (Barc). 45:483-496.

9. Kress HG (2010) Tapentadol and its two mechanisms of action: is there a new pharmacological class of centrally-acting analgesics on the horizon? Eur J Pain 14: 781-783.

10. Schröder W, Vry JD, Tzschentke TM, Jahnel U, Christoph T (2010) Differential contribution of opioid and noradrenergic mechanisms of tapentadol in rat models of nociceptive and neuropathic pain. Eur J Pain 14: 814-821.

11. Afilalo M, Morlion B (2013) Efficacy of tapentadol ER for managing moderate to severe chronic pain. Pain Physician 16: 27-40.

12. Adivelu N, Timchenko A, Huang Y, Sinatra R (2011) Tapentadol extendedrelease for treatment of chronic pain: a review. J Pain Res 4:211-218.

13. Vincenzo Pota, ManlioBarbarisi, Pasquale Sansone, Marco Moraci, Maria Caterina Pace, et al. (2012) Combination therapy with transdermal buprenorphine and pregabalin for chronic low back pain. Pain Manag 2:23-31.

14. Attal N, Cruccu G, Baron R, Haanpää M, Hansson P, et al. (2010) EFNS guidelines on the pharmacological treatment of neuropathic pain: 2010 revision. Eur J Neurol 17: 1113-1113e88. 